\title{
TRAS LOS PASOS DE WASHINGTON IRVING: VIAJERAS NORTEAMERICANAS EN LA ANDALUCÍA DEL SIGLO XIX*
}

\author{
Blasina Cantizano Márquez \\ Universidad de Almería
}

\section{RESUMEN}

Dentro de la literatura de viajes sobre España, The Alhambra (1832) de Washington Irving supuso el acercamiento de la historia y la cultura españolas no solo a la población norteamericana coetánea, sino a multitud de lectores. Sus relatos contribuyeron de gran manera a la creación y difusión de la imagen romántica de España: un país algo atrasado poblado por toreros, bandoleros y mujeres de exótica belleza. Partiendo de esta visión particular, muchos son los viajeros norteamericanos que posteriormente siguen sus pasos y visitan los lugares mencionados en su libro. El presente artículo se centra en dos viajeras norteamericanas que visitan Andalucía en la segunda mitad de siglo: Susan Hale y Katharine Lee Bates. No solo ofrece un acercamiento a sus biografías y las circunstancias particulares de sus viajes al sur de la Península, sino que también aporta un análisis de las obras surgidas durante o tras su estancia en España, y más concretamente en Andalucía, con la intención de comprobar si esta literatura de viajes femenina sigue la misma línea de los viajeros románticos o, por el contrario, supone un punto de vista diferente al masculino tradicional.

Palabras clave: literatura de viajes, viajeras norteamericanas, Andalucía, Susan Hale, Katharine Lee Bates.

\section{FOLLOWING WASHINGTON IRVING'S STEPS: AMERICAN WOMEN TRAVELLERS IN ANDALUSIA IN THE NINETEENTH CENTURY}

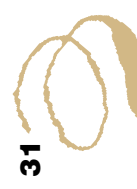

\section{Abstract}

In relation to travel literature about Spain, Washington Irving's The Alhambra (1832) meant an authentic approach to Spanish history and culture not only to the American people of his time, but also to general readers worldwide. The tales included in his book contributed to the creation and promotion of the Romantic image of Spain: an old-fashioned country inhabited by bullfighters, bandits and beautiful, exotic women. From this particular perspective, many American travel writers visited Spain following Irving's footsteps and paying attention to his books and advice. This paper will focus on two American women writers who travelled to Andalusia during the second half of the nineteenth century: Susan Hale and Katharine Lee Bates. The paper will not only offer an approach to their biographies and particular travels to the South of Spain, but will also provide an analysis of the texts produced after or during their travels. It is our purpose to establish a comparison between travel books written by men and those written by women, in order to prove whether the female experience provides a different perspective to the traditional one offered by men.

KeYwords: travel literature, American women travellers, Andalusia, Susan Hale, Katharine Lee Bates. 
La Guerra de la Independencia (1808-1814) y el Romanticismo fueron factores decisivos en la afluencia de viajeros extranjeros a Espańa, un país que había quedado fuera del "Grand Tour» turístico y formativo de la burguesía europea del XVIII. Todo lo que había sido tachado de atraso y barbarismo con anterioridad supone ahora, bajo la ideología romántica, un campo de reflexión y aventura que llevará a un sincero interés por España y su población. Stanley G. Payne es así de rotundo al respecto:

Si el Romanticismo era la forma cultural de la modernidad, Espańa era del todo moderna y podía ser la maestra de Europa. De este modo se superaban los términos de desprecio que con tanta frecuencia se encontraban en los textos extranjeros, al tiempo que la cultura española, original y de alta calidad, se ofrecía al mundo (Payne 2017: 28).

Ya entrado el siglo XIx, se produce una masiva afluencia de viajeros extranjeros que, por motivación concreta o mero placer, recorren la Península con la intención de encontrar lo particular, lo exótico y diferente al resto del mundo occidental. La cultura y la estética musulmanas adquieren ahora un destacado protagonismo, el pasado de Al-Andalus una motivación ańadida para viajar al Sur y descubrir la huella árabe en su sociedad. Es importante destacar que «la imagen que se tiene en el mundo de España será la romántica y, como personificación más representativa de ella y de lo español, Andalucía y lo andaluz» (Muñoz 1981: 17).

Los libros de viaje, considerados de carácter inferior, permitieron a muchas mujeres convertirse en sujetos artísticos de primer nivel sin necesidad de sentirse infravaloradas. Alberto Egea afirma que este género literario «fue elegido de manera consciente y cuidadosa, ya que escribir como sociólogas, naturalistas, cartógrafas o antropólogas hubiera sido casi imposible debido a que estos eran discursos reservados casi exclusivamente al varón» (2009: 25). Del colectivo femenino norteamericano que recorre la Península Ibérica en el siglo xIx, destacamos a Susan Hale (1833-1910), Louise Chandler-Moulton (1835-1908), Merrydelle Hoyt (1860-1933), Elizabeth W. Champney (1850-1922) y Katharine Lee Bates (1859-1929). Todas ellas comparten una serie de rasgos comunes que facilitarán el viaje literario: proceden de una posición social acomodada y por ello no se deben a las exigencias de ninguna editorial, una situación ventajosa que también les permite ser más libres a la hora de elegir destino y recorrer el territorio. Pese a que «el viajero, cuando recorre otro país, lo hace a sabiendas de que transporta consigo un pesado bagaje de prejuicios que, al fin y al cabo, son parte integrante de su identidad como ciudadanos naturales de otro país» (López y Rivas 2013: vii-viii), estas miradas femeninas se ofrecen para complementar y contrastar las ofrecidas por reputados viajeros e hispanistas. La mirada femenina, según Lady Eastlake, era necesaria en los relatos de viajes y así lo expresó

* Este trabajo se incluye dentro del proyecto CEI-Patrimonio, en el que participa el Grupo de Investigación Lindisfarne (HUM807) de la Universidad de Almería. 
en un artículo anónimo publicado en la Quarterly Review de Londres: «But, in truth, every country with any pretensions to civilization has a twofold aspect, addressed to two different modes of perception, [...] a home life as well as a public life, and the first quite necessary to interpret the last. Every country, therefore, to be fairly understood requires reporters of both sexes» (1845: 99). Igual que otras mujeres de la época tardovictoriana, con sus escritos de viajes Susan Hale y Katharine Lee Bates asumieron plenamente el papel de «reporters» de lo espańol, demostrando a su vez que podían abandonar el espacio doméstico para hacerse oír en el espacio público.

\section{WASHINGTON IRVING EN ESPAÑA}

Uno de los mayores responsables de la creación y difusión de la imagen romántica de Espańa es el norteamericano Washington Irving (1783-1859), conocido por ser el autor de The Alhambra (1832). Su primer viaje a Espańa tiene lugar durante los años 1826 y 1829 , periodo que dedicó no solo a viajar por el país sino también a estudiar historia, lengua y literatura, además de investigar en el Archivo de Indias, pues traía como encargo realizar un libro sobre los viajes de Cristóbal Colón, empresa que luego da lugar a varias obras sobre este periodo histórico: The Life and Voyages of Christopher Columbus (1827), A Chronicle of the Conquest of Granada (1829) y Voyages and Discoveries of the Companions of Columbus (1931). A diferencia de otros viajeros extranjeros, interesados en rutas, geografía, historia y vivencias personales, "Washington Irving busca lo maravilloso, lo mítico, lo fantástico y, como romántico, intenta captar la esencia del carácter del pueblo» (García-Montón y García-Romeral 2000: 264). Su visita a Andalucía, y más concretamente a Granada, se convierte entonces en una estancia prolongada y una experiencia única en su faceta como escritor. Alojado en el palacio de la Alhambra, y con la colaboración de lugareños como la tía Antonia o Mateo Ximénez, se dedica no solo a describir la ciudad que recorre sino también, y más importante, a recopilar antiguas leyendas de otros tiempos, historias de musulmanes y cristianos, de espíritus y personas reales que le convierten en cronista de la ciudad para la posteridad. Durante los ańos 1842 y 1846 ejerce de embajador norteamericano en Madrid, ampliando su conocimiento del país con otra serie de viajes.

Por la calidad y variedad de sus obras de tema español, Irving puede ser considerado como uno de los primeros hispanistas norteamericanos y una referencia obligada para una aproximación a la historia y la cultura españolas. La repercusión de sus obras contribuyó a la afluencia de viajeros extranjeros a España, y más concretamente a Andalucía, motivados no ya por las leyendas fantásticas que recoge en su conocido Cuentos de la Alhambra, sino también por afirmaciones tan rotundas como la siguiente: «There is something, too, in the sternly simple features of the Spanish landscape, that impresses on the soul a feeling of sublimity» (1906: 4). La huella de sus experiencias en Espańa contribuirá en gran manera a la creación y difusión de una imagen romántica de la que cuesta desprenderse, «bien sea por escasez de otras fuentes de información o por el enorme éxito que cultivó, generará la imagen arquetípica de Espańa en el imaginario colectivo norteamericano que, 
con variantes, se mantendrá hasta bien entrado el siglo XX» (Herrero 2016). Sin lugar a dudas, tal como afirma Celia Wallhead, «Irving's epic journey and his highly successful writings on the subject put Andalusia con the map, and Spain in general has been eternally grateful ever since» (2008: 13). Son muchos los norteamericanos que todavía hoy, en el siglo XXI, siguen sus pasos por España.

\section{SUSAN HALE (1833-1910)}

Nacida en Boston en el seno de una familia numerosa, acomodada y de gustos refinados, Susan Hale creció y se educó en un ambiente que favorecía la creación artística e intelectual: su padre era abogado y editor del Boston Daily Advertiser, su madre, escritora y traductora. No es de extrañar, por tanto, que varios de sus hijos ejercieran posteriormente actividades relacionadas con la pintura y la escritura, siendo su hermano Edward Everett el más reconocido de todos. La vida personal de Susan Hale no fue convencional para su época, puesto que no se casó, ni se dedicó a las labores propias de una dama de su posición social. Las dificultades económicas que experimentó la familia la condujeron durante unos años al mundo de la enseñanza. Además de actividades físicas al aire libre y su pasión por los viajes (a Tierra Santa, Europa, Egipto, Sudamérica, etc.), la vida profesional de esta autora se orientó a la creación artística, combinando literatura y pintura en obras muy diversas en cuanto a contenido y formato: libros de viaje, ficción, monólogos, e incluso ejerció de actriz en alguna obra, mientras que sus acuarelas se exhibieron en Boston y Nueva York con muy buena acogida.

Mujer de espíritu decidido e independiente, había realizado viajes en solitario, pero en su visita a España en 1882, viaja acompañada de su hermano Edward, su sobrina y una amiga de la familia, Mary Marquand. El hecho de viajar en grupo era una costumbre bien extendida entre las mujeres de cierta posición, puesto que «for women travelling alone, travel has always been and still is difficult, and to present oneself as essentially being alone invites questions about traveler's morals» (Harper 2001: 17). De este viaje familiar surgen dos obras bien distintas: A Family Flight Through Spain (1883), de la autora, y Seven Spanish Cities and the Way to Them (1883), de su hermano Edward, que varían en estilo, contenido y perspectiva. Este último puede considerarse de corte más tradicional. Susan, por el contrario, «decide escribir un relato de viajes novelado sirviéndose de las licencias que le permite un género híbrido» (De la Torre 2010: 255) y así combina realidad y ficción, alejándose del formato tradicional de los libros de viaje repletos de datos históricos, valoraciones artísticas y descripciones tediosas.

Aunque se trata de una viajera culta y documentada y el periodo de su viaje coincide con el auge de los movimientos realista y costumbrista, Susan Hale recurre a clichés románticos en muchas de sus descripciones. Así, se deja llevar por reminiscencias de los viajeros románticos en sus comentarios sobre Andalucía: «In short it was Andalusia! Andalusia! The land of romance and sunshine, the most beautiful province of beautiful Spain» (1899: 145). Esta influencia parece extenderse también a su vida privada, ya que en una carta personal escrita en junio de 1882 
a su hermana Lucrecia describe Granada desde una perspectiva orientalista: «But, oh, my dear! We have reached the heavenly culmination of our trip, -for Granada is the dream of Aladdin's lamp,- and a lovely place to be in for June» (1921: 134). Pese a esta fascinación por el exotismo y el aire oriental de la ciudad, Hale viaja a finales del siglo xix y es consciente de los cambios que se han producido en la ciudad desde que Irving la visitase, ofreciendo algún comentario muy acertado sobre el incipiente turismo de masas que invade las estancias de la Alhambra, por ejemplo, que ansiaba «the experience of Mr. Irving, who lived in rooms actually within the palace precincts. He had the place all to himself, and learned to know it before the great band of tourists had invaded the spot, or guide books, with their convenient gossip, laid bare the secrets of the Alhambra» (Hale 1899: 243-244). Tales quejas, si por una parte servían para lamentar las molestias derivadas de una presencia excesiva de visitantes, por otra no hacían más que perpetuar la memoria y la leyenda del escritor estadounidense cuyos pasos tantos compatriotas suyos más o menos fielmente seguían.

Además de recorrer otras zonas del Mediterráneo y Sudamérica en fechas posteriores, Susan Hale incluyó de nuevo España entre sus rutas en dos ocasiones más (1883 y 1887) para tener la posibilidad de vivir nuevas experiencias. Una de las actividades a las que no se resisten los viajeros extranjeros que visitan España es la de asistir a festejos taurinos, pues recordemos que «las corridas alcanzan su plena madurez durante el segundo cuarto del siglo XIX, ya con la forma que ha llegado a nuestros días» (Payne 2017: 29). Susan Hale no puede ser menos y, pese a ser una mujer atrevida, no deja de escandalizarse ante la crueldad de la que es testigo en una corrida en Sevilla durante la feria de abril de 1887. Aun así, asegura que le ha servido para evitar que otros lo hagan: «Yes, $M e$, at the bullfight! It was perfectly horrible, sickening, disgusting [...] On the whole it is just as well, because now I can use all my powers of speech to exhort others not to go" (Hale 1921: 182). La autora refleja en este breve comentario la conciencia reformista de muchas mujeres estadounidenses del momento, que aprovechaban sus escritos de viajes para incidir en debates de toda índole, incluidos los animalistas.

\subsection{A Family FLIGHT Through Spain (1883)}

La intención de Susan Hale con este libro es, sin duda, informar a la vez que entretener al lector; es por ello que, más que un libro autobiográfico sobre su propia experiencia, este se organiza en torno a la familia Horner, sus cuatro hijos y la señorita Lejeune, en un viaje de inmersión cultural por España. Con este formato, la autora combina sus propias experiencias con aquellas que plantea para sus personajes, la descripción de paisajes y población con la ficción, en una obra que se aleja de anteriores libros de viajes, como puntualiza la propia autora al lector de su obra: "Descriptions of Cathedrals are always tedious to those who have not seen them, and it will not do to weary the reader with a detailed account of all the Horners visited» (Hale 1883: 47). 
La narración comienza en primavera, con la llegada de estos viajeros al País Vasco a través de Irún. La ruta atraviesa posteriormente la meseta (Burgos, Madrid, Toledo) para detenerse en Andalucía (Cádiz, Sevilla, Granada, Córdoba) y finalizar el viaje en Zaragoza y Barcelona. En los primeros capítulos, se resalta el contraste entre Espańa y Francia, además de ofrecer datos históricos, etnográficos y artísticos que sitúan al lector ante un escenario real en el que se mueven sus personajes de ficción. En este sentido, resulta curioso el capítulo III, en el que la familia Horner practica el idioma y se documenta sobre el país leyendo guías de viaje en el trayecto del tren a Burgos: "The guide-books kept our party well posted on the points of interest, historic and romantic, and they would have been glad to pause often to make a sketch or inspect a castle» (1883: 29). También el hecho de que se adapten a las costumbres culinarias del país y se atrevan con una amplia variedad de productos y elaboraciones como el puchero o los churros que degustan en el capítulo IV: «Next morning instead of coffee, there was brought to their rooms a tray containing cups of thick chocolate, and bread, with a tumbler full of water for each person, and resting across the tumbler a long piece of crisp white sugar, called azucarillo» (1883: 35).

A lo largo de las páginas del libro, el lector descubre que la señorita Lejeune parece ser la verdadera organizadora del viaje a Espańa: su interés personal se traslada al pasado, cuando visitaba a una familiar casada con un explorador español y quedaba embelesada por las curiosidades y los souvenirs de aquella casa (1883: 35). Esta anécdota de ficción está relacionada con la vida real de los hermanos Hale, puesto que Edward también hace referencia a estos recuerdos infantiles en la introducción de su libro: «Their letters from Spain and their Spanish curiosities were among the home excitements of my childhood; and the great red-letter day was the day of their return. Well do I recollect the box of bon-bons they brought me» (1883: iii).

En Andalucía, la estancia de los viajeros es de mayor duración, se detienen en varias ciudades con la intención de profundizar en la cultura e idiosincrasia de una región cargada de historia. Por los comentarios de la narradora, el sur es descrito como un lugar idílico superior al resto de España:

The wines and olives of Andalusia, its grapes, and oranges, and fruits of all kinds, are the finest, its horses and cattle are the best, its bulls are the fiercest of all Spain. Its cities are famous for their attractions, and its men and women for their grace and beauty. All things take on an air of loveliness in this land of warmth and glow (1883: 152).

Sin lugar a dudas, las descripciones e impresiones de los personajes no hacen sino transmitir el peso de la tradición romántica y el gusto por la estética oriental de muchos viajeros extranjeros anteriores, «la exaltación hiperbólica de la belleza de la tierra andaluza no constituye nada nuevo, ya que ésa es una actitud ampliamente compartida por andaluces y extranjeros» (Bernal 1985: 18). Hale refuerza esta visión orientalista de Andalucía con el uso de escenas y la elección de adjetivos que ofrecen una imagen estereotipada. Así, la señorita Lejeune y el señor Horner intercambian opiniones similares sobre el paisaje de Córdoba:

«How Eastern! Is it not?» -exclaimed Mr. Horner.

«More Eastern than the East»-replied Mrs. Lejeune (1883: 137). 
Igual ocurre cuando visitan Granada y describen el entorno de la Alhambra. Las impresiones y comentarios de la narradora parecen estar guiados por la vida y obra de Washington Irving cuando menciona a la tía Antonia de los cuentos (1883: 251) y su versión de la conquista de la ciudad a manos de los Reyes Católicos: «Every one has read the story of the surrender in Washington Irving's Conquest of Granada. The besieged city was suffering the distress of the famine. Autumn arrived, a rigorous winter was approaching; the people sank into deep despondency» (1883: 229). Las referencias a tan ilustre predecesor no se limitan a Andalucía, puesto que cuando los Horner llegan a Barcelona también hacen referencia al Irving menos conocido, el diplomático que tuvo contacto con políticos, aristocracia y realeza. Recuerdan y comparten sus palabras sobre Barcelona: "Washington Irving had occasion to visit it while he was American minister in Spain [...] He was delighted with the city, in contrast to the less favoured situation of Madrid, and describes living there as the poetry of existence» (1883: 353).

Sin duda, Susan Hale fue una mujer bien documentada y preparada para el viaje, al que se enfrentaba con nociones de historia, literatura e idiomas para conseguir el máximo partido de la experiencia. Una mujer intrépida, decidida y algo arriesgada, «otra de tantas mujeres adelantadas a su tiempo, que abrió el camino de la emancipación y de la lucha por la igualdad simplemente con su ejemplo de vida y con el testimonio que dejó en sus obras literarias» (De la Torre 2010: 255).

\section{KATHARINE LEE BATES (1859-1929)}

En Estados Unidos Katharine Lee Bates es bien conocida por ser la autora de "America the Beautiful», un poema que escribió en 1893, revisó posteriormente para The Boston Evening Transcript (1904) y cuya versión final de 1913 es más extensa y se acompaña de música, lo que lo convierte en una exaltación de América, además de ser considerado por muchos el verdadero himno del país. Nacida en Massachusetts, estudió y se graduó en 1880 en el prestigioso Wellesley College, centro precursor del movimiento feminista. Trabajó algunos ańos como profesora de inglés, para posteriormente volver a Wellesley y continuar su carrera académica. Allí ejerció de profesora de literatura en el departamento de inglés e incluso llegó a ser directora del mismo hasta su jubilación. A lo largo de su carrera profesional desarrolló una intensa labor investigadora, docente y literaria. Es importante también el hecho de que fuera lesbiana y mantuviera una relación larga y estable con otra compañera del departamento de inglés, Katharine Coman; es por este motivo que «tanto grupos feministas como activistas lesbianas reclaman su figura como una representante importante de estos movimientos» (Egea 2009: 54). Como autora literaria destacamos su prolífica producción: libros de poemas, literatura infantil y escritura de viajes, inspirada en sus experiencias por Europa y Oriente Medio.

A España viaja en 1899, un año después de que el país se enfrentara a Estados Unidos en la Guerra de Cuba y las Filipinas. El contraste entre una mujer reivindicativa e independiente y la conservadora sociedad española de la época es interesante, porque, pese a lo inusual de la situación, pudo conectar y adaptarse bien 
al lugar que visitaba. De sus experiencias en España surge Spanish Highways and Byways (1900) y también, aunque menos conocido, su relato para niños In Sunny Spain with Pilarica and Rafael (1913), en el que recoge multitud de historias, canciones tradicionales y versos originales, los traduce y adapta en una obra de ficción dirigida a jóvenes lectores.

\subsection{SPANISH HIGHWAYS AND BYWAYS (1900)}

Ya desde la primera página, la autora advierte al lector que su libro es «nothing more than a record of impressions» (1900). Del modo más tradicional, el relato se escribe en primera persona y se articula en torno al recorrido del viaje. La novedad reside en que Katharine Lee Bates aporta una visión más objetiva y crítica que muchos de sus predecesores, pues no solo ofrece descripciones e impresiones personales de los lugares que visita, sino que también incluye referencias históricas y artísticas e incluso inserta poemas populares y de autores clásicos. El viaje lo realiza acompañada de una joven compatriota que estudia arte en París: lo inician en el País Vasco, recorren Castilla y Andalucía, región a la que dedica la mayor parte del libro, para terminar abandonando la Península por Galicia.

En su libro de viajes sobre España, Bates, además de contrastar e incluir referencias bien documentadas, se interesa por la población que conoce, por su modo de vida, por las dificultades a las que se enfrentan en un país que intenta recuperarse de una derrota internacional importante. El primer capítulo del libro, que dedica a romper con estereotipos negativos sobre la población, lleva por título "The Lazy Spaniard», algo que contradice y rebate a lo largo de sus primeras páginas con ejemplos como la vida diaria de una maestra de pueblo:

This 'lazy Spaniard', however, not only keeps her Kindergarten well in hand from nine to twelve, but instructs the same restless mites [...] in reading and counting from two to four, gives a Spanish lesson from six to seven and struggles with the pathetic ignorance of grown men and women in the night school from eight to half-past nine or ten (1900: 8).

En Granada intenta alejarse de las meras descripciones de la Alhambra y opta por una aproximación más actual no ya a la fortaleza nazarí, sino también a la ciudad, dedicando un capítulo a las honras fúnebres que se organizan en honor al escritor y diplomático Ángel Ganivet (1865-1899): «I was specially touched in Granada by the whole-souled sympathy and veneration with which the city rendered public honor to one of its sons, Angel Ganivet, who died in the preceeding winter, a poet hardly thirty» (1900: 39). Durante su mes de estancia en la ciudad ofrece algunas impresiones preciosistas del lugar y elogia la estética musulmana, pero también se muestra crítica con la explotación del entorno: «The habit of begging, that plague of tourist resorts, is an incessant nuisance on the Alhambra hills» (1900: 30).

Mujer coherente con sus principios, acepta asistir a una corrida de toros en Sevilla para comprobar si realmente era un espectáculo tan cruel como suponía, 
«I have a prejudice against being prejudiced, and to the bullfight I went» (1900: 120). De esta experiencia surge el capítulo IX, «A Bull-fight», que se inicia con una revisión histórica de este entretenimiento nacional e incluye referencias concretas a modas, ambiente y toreros de la época. A modo más personal, ofrece una detallada y sangrienta descripción de los seis toros de la corrida, combinada con impresiones muy directas y comentarios cargados de ira contra el espectáculo: «I was long past indignation, past any acuteness of pain, simple sickened through body and soul and unutterably wearied with this hideous monotony of slaughter» (1900: 130).

En su testimonio de viajes, además de referencias históricas y literarias del pasado, es importante destacar que aporta información sobre la vida de una España de entre siglos, camino a la modernidad. Además de cuestiones sobre política, también dedica algunos párrafos a denunciar la situación de la mujer en España, destacando sobre todo su desprotección social: «A young Spanish girl cannot walk alone, however sedately, in Seville, without a running fire of salutations [...] And even in Madrid, Spanish girls of my acquaintance have broken their fans across the faces of men who tried to catch a kiss in passing» (1900: 189). En este y otros casos, los comentarios de Bates, al igual que los de Susan Hale, no hacen más que poner de relieve la conciencia social de muchas mujeres de Nueva Inglaterra y que a menudo convirtieron los libros de viajes en un útil y potente altavoz para sus reivindicaciones.

Lo relevante de este libro es que su autora consigue distanciarse de la conocida imagen de la España romántica difundida por la literatura anterior. Tal como observa Alberto Egea, «a pesar de las ideas preconcebidas llenas de tópicos y estereotipos que la autora traía a su llegada a España, a medida que va conociendo a los andaluces y su cultura consigue cambiar sus apreciaciones por otras menos esencialistas» (2009: 53). Aunque no deja de caer en las descripciones pintorescas y preciosistas de viajeros anteriores, durante su visita a Sevilla la propia Bates conviene que «[i]t was all as Oriental as a dream» (1900: 51). La autora es testigo de una realidad concreta y a ella se debe; es por este motivo que su relato está cargado de referencias al desastre español en Cuba y a las diferentes reacciones de la población. En una ocasión, por ejemplo, habla sobre «the bronze statue of Columbus which the women of Granada had recently stoned because, by discovering America, he brought all the Cuban problems upon Spain» (1900: 26). También en Granada recoge la opinión de un joven español sobre el enfrentamiento entre Espańa y Estados Unidos de una manera directa y clara: "America is the richest country of all the world. When America fought us it was as a rich man, fed and clothed, fighting a poor man weak from famine. And the rich man took from the poor man all that he had. Spain had nothing left -nothing!» (1900: 34).

Bates, en suma, no rehúye ninguno de los temas esenciales que, según ella, afectaban a los españoles, siendo las consecuencias de la guerra uno de los más dolorosos. A modo de valoración general, Alberto Egea concluye que Katharine Lee Bates «acierta en su análisis de una complicada situación derivada de la derrota española de 1898 ante el recién nacido gigante norteamericano, apostando por un nuevo espíritu de concordia que pusiese fin a las asperezas» (Egea 2009: 53). Siguiendo los pasos de Washington Irving varias décadas atrás, a través de sus escritos de viajes Bates se 
convertiría en una especie de figura mediadora, tendiendo de nuevo puentes entre las culturas hispánica y anglosajona en los albores de un nuevo siglo.

\subsection{In SunNy Spain With PILARICA and RafaEL (1913)}

Durante su viaje, Katharine Bates no olvida su faceta de escritora para niños y recopila importantes testimonios de la tradición oral española. Muchos ya aparecen recogidos en el capítulo xx de su libro de viajes, titulado "Coral Games of Spanish Children", en el que reproduce canciones y juegos que oye en las calles españolas y establece comparaciones con los que existen al otro lado del Atlántico, tanto en Estados Unidos como en las antiguas colonias espańolas. El libro comienza con una carta de la escritora a sus jóvenes lectores, de ahí el lenguaje claro y sencillo que utiliza. Es aquí donde Katharine Lee Bates se justifica y explica la razón de este libro infantil sobre España: la necesidad de acercar fronteras, motivando también a sus lectores a que hagan sus propios descubrimientos: "Do American children play as many games as Spanish children play? Do they know as many riddles? Can they sing as many merry songs? Perhaps not» (1913: xiv). En esta carta explica también que el padre de Pilar y Rafael está lejos de su familia porque se encuentra en Cuba, defendiendo la isla contra los invasores norteamericanos. Su intención es, una vez más, tender puentes entre dos países, dos culturas que se encuentran en una situación de desencuentro y enemistad.

De la mano de los pequeños Pilar y Rafael, la trama de In Sunny Spain with Pilarica and Rafael tiene mucho que ver con los Cuentos de la Alhambra de Washington Irving, con la magia, lo inesperado y las historias de tradición oral. En ambos aparece la figura de un narrador, conocedor de misterios y secretos, que hila relatos fantásticos con sucesos reales. Y en ambos casos la historia tiene lugar en el entorno de la Alhambra: "Grandfather did not have to think long, for he was the wisest man in Spain, as all the children on the Alhambra hill would have told you, and he knew more rhymes and riddles than all the professors in all the universities and all the preachers in all the pulpits put together» (1913: 4). La tía Antonia fue la particular fuente de información de Washington Irving, mientras que Bates crea la figura del abuelo de los protagonistas, un anciano y sabio narrador que se adapta a las necesidades de los niños:

«Grandfather tells me many more stories than he tells you», boasted Rafael. «You are all for riddles and verses, but he and I talk together, like men, of the affairs of the world. Juan Cigarron, who lived a long, long while ago, before you, and even I, had been born, made believe that he was a great magician and could see anything, even if it was hidden in the very depths of the earth, unless, to be sure, there was a blue cloth wrapped around it» (Bates 1913: 12).

En este libro, además del relato de ficción, la autora recoge versos, acertijos y canciones de corro tan conocidas como El señor don gato o El patio de mi casa, que ella misma traduce al inglés de la siguiente manera: 
The garden of our house it is

The funniest garden yet,

For when it rains and rains and rains,

The garden it is wet.

And now we bow,

Skip back and then advance,

For who know how

To make a bow

Know how to dance [...] (1913: 26).

El contraste de estas dos obras de tema español, de contenido y enfoque tan distinto, demuestra la capacidad creadora de Katharine Lee Bates. Dirigidas a dos públicos bien diferentes y respondiendo a sus necesidades particulares, estas obras pueden leerse de forma individual pero también complementaria no solo para constatar el influjo de Irving sino también para tener una visión clara y de conjunto de la sociedad española de finales de siglo XIX.

\section{CONCLUSIONES}

El viaje al extranjero de estas autoras coincide no ya con un periodo de expansión imperial de Estados Unidos, sino con un momento de mayor protagonismo e independencia de la mujer en los ámbitos artístico e intelectual. A diferencia de las primeras viajeras europeas, que viajan como parte de un viaje familiar acompañando al esposo en un recorrido específico, estas viajeras norteamericanas viajan por iniciativa propia, con un interés o motivación particular que las lleva a emprender el camino. Son mujeres de posición social acomodada, que gozan de una educación privilegiada y se preocupan en documentarse en la historia, la literatura, el arte o los idiomas de los lugares que visitan. Son viajeras cultas y profesionales, que escriben para un público determinado al que en ocasiones se dirigen directamente, explicando o justificando el contenido, enfoque o particularidades de sus libros. Aunque ellas mismas aseguran que son obras muy personales, cargadas de impresiones y comentarios propios, estos libros deben leerse con atención, como contraste y complemento de otras obras escritas por viajeros canónicos de reconocido prestigio. Solo así podrá completarse la imagen que proyectaba España en el siglo xix fuera de sus fronteras.

ReCibido: junio de 2018; ACEPTADo: octubre de 2018. 


\section{BIBLIOGRAFÍA}

Bates, Katharine Lee (1900): Spanish Highways and Byways, Londres: Macmillan.

BAtes, Katharine Lee (1913): In Sunny Spain with Pilarica and Rafael, Londres: J.M. Dent.

Bernal Rodríguez, Manuel (1985): La Andalucía de los libros de viaje del siglo XIX, Sevilla: Editoriales andaluzas unidas.

Champney, Elizabeth (1883): Three Vassar Girls Abroad. Rambles of Three College Girls on a Vacation Trip through France and Spain for Amusement and Instruction. With their Haps and Mishaps, Boston: Estes and Lauriat.

De la Torre Laviana, María (2010): «Susan Hale: Una viajera en el camino hacia la emancipación», Odisea 11: 249-257.

Eastlake, Elizabeth Rigby (1845): «Lady Travellers», Quarterly Review 76 (June): 98-137.

Egea Fernández-Montesinos, Alberto (coord.) (2009): Viajeras anglosajonas en España. Una antología, Sevilla: Centro de Estudios Andaluces.

García-Montón, Isabel y Carlos García-Romeral (2000): «Viajeros americanos en Andalucía durante los siglos XIX y XX", Revista Complutense de Historia de América 26: 261-279.

Hale, Edward (1883): Seven Spanish Cities and the Way to Them, Boston: Roberts Brothers.

Hale, Susan (1883): A Family Flight through Spain, Boston: Lothrop Publishing Company.

Hale, Susan (1899): Young Americans in Spain, Boston: Lothrop Publishing Company.

Hale, Susan (1921): Letters of Susan Hale (1833-1910), Boston: Marshall Jones Co.

Harper, Lila M. (2001): Solitary Travelers: Nineteenth-Century Women's Travel Narratives and the Scientific Vocation, Madison, NJ: Fairleigh Dickinson University Press.

Herrero, Carlos (2016): «La España inventada de Washington Irving», Diálogo Atlántico 5. URL: http://dialogoatlantico.com/2016/05/la-espana-inventada-de-washington-irving/; $14 / 05 / 2018$.

Hoyт, Merrydelle (1914): Mediterranean Idylls, Londres: Duckworth.

IRving, Washington (1832): The Alhambra, Londres: Colburn and Bentley.

Irving, Washington (1906): Tales of the Alhambra, Londres: Macmillan and Co.

López Folgado, Vicente y María del Mar Rivas Carmona (eds.) (2013): La Andalucía rural vista por viajeros extranjeros. Campos, posadas y tabernas, New York: Peter Lang.

Moulton, Louise Chandler (1897): Lazy Tours in Spain and Elsewhere, Boston: Roberts Brothers.

Muñoz Rojas, José A. et al. (1981): La imagen romántica de España, Madrid: Ministerio de Cultura.

Payne, Stanley G. (2017): En defensa de España. Desmontando mitos y leyendas, Barcelona: Espasa.

Wallhead, Celia (2008): Washington Irving and Spain. The Romantic Movement, the Relcreation of Islamic Andalusia and the Critical Reception, Bethesda, Dublin, Palo Alto: Academica Press. 